Supporting Information for

\title{
Revisiting the origins of the fracture energy of tough double-network hydrogels with quantitative mechanochemical characterization of the damage zone
}

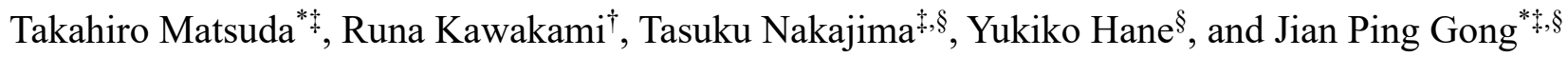 \\ *Faculty of Advanced Life Science, ${ }^{\dagger}$ Graduate School of Life Science, ${ }^{\S}$ Institute for Chemical Reaction Design and \\ Discovery (WPI-ICReDD); Hokkaido University, Japan \\ E-mail: gong@sci.hokudai.ac.jp; Address: N21W11, Kita-ku, Sapporo 001-0021, Japan.
}

\section{Contents}

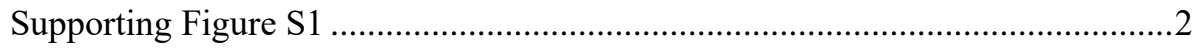

Supporting Note I (and Figures S2-S5): Estimation of the intrinsic fracture

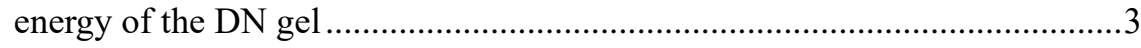

Supporting Note II: Potential errors in the fluorescence method using mechanoradical polymerization .10

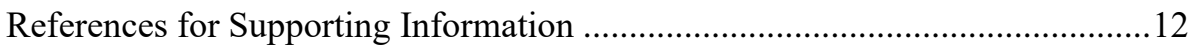




\section{Supporting Figure S1}

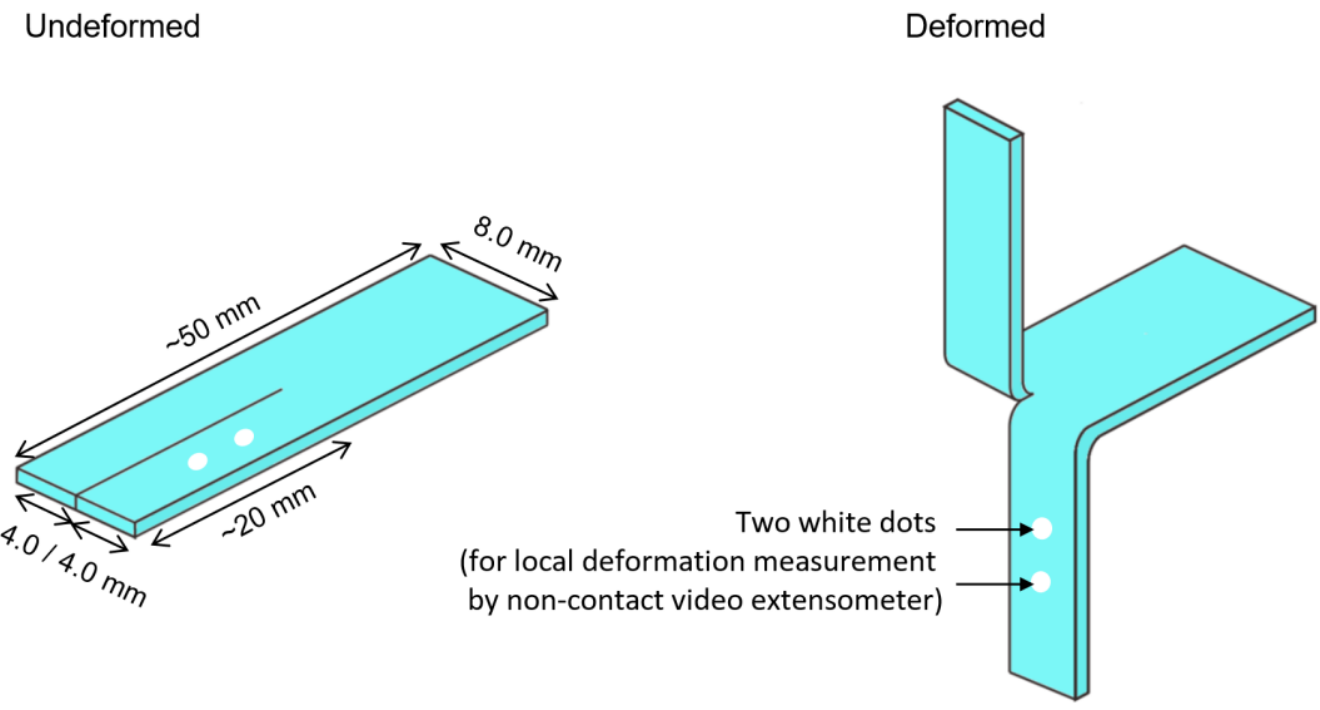

Figure S1. Experimental setup of the fracture test with a trouser-shaped specimen. Elongation ratio $\lambda$ of the lower leg was measured using a non-contact video extensometer. 


\section{Supporting Note I (and Figures S2-S5): \\ Estimation of the intrinsic fracture energy of the DN gel}

The fracture energy of a pre-stretched DN gel was characterized to estimate the intrinsic fracture energy $\Gamma_{0}$, which corresponds to the fracture energy of the crack tip in the damage zone, whereby the first network is significantly broken in the second network matrix. In this experiment, we measured the energy release rate at the onset of the crack propagation, $G_{\mathrm{c}}$, using a single-edge notch test (Figures S2b and S3a). ${ }^{\mathrm{S} 1, \mathrm{~S} 2}$ The $G_{\mathrm{c}}$ of the virgin DN gel and the corresponding PAAm single-network gel were also measured for comparison. We used dumbbell-shaped samples (standardized to JIS-K 6251-6; $25 \mathrm{~mm}$ length, $4 \mathrm{~mm}$ width, and 1.0-1.7 thickness; Figure S2a) because this shape is appropriate for inducing internal fracturing with the first stretching. The fracture energy $G_{\mathrm{c}}$ of the DN gel was characterized as:

$$
G_{\mathrm{c}}=2 k W_{\mathrm{c}} c_{0}=\frac{6 W_{\mathrm{c}} c_{0}}{\sqrt{\lambda_{\mathrm{c}}}}
$$

where $c_{0}$ is the notch length, $\lambda_{\mathrm{c}}$ and $W_{\mathrm{c}}$ are the critical elongation ratio and critical strain energy density, respectively, at which crack propagation starts upon extension, and $k$ is the prefactor that depends on the elongation ratio. We assumed $k=$ $3 \lambda^{-1 / 2}$, which is commonly used in recent studies. ${ }^{\mathrm{S}-\mathrm{S} 8}$ Note that the parameter $k=3 \lambda_{\mathrm{c}}{ }^{-1 / 2}$ is an empirical factor that is valid for some rubbers at $\lambda_{\mathrm{c}}<3,{ }^{\mathrm{S} 2}$ and roughly valid for some rubbers at $\lambda_{\mathrm{c}}<5 .{ }^{\mathrm{S} 9}, \mathrm{~S} 10$ The notch $(\sim 1.0 \mathrm{~mm})$ was made using a razor blade, and its length was individually measured with a caliper $\left(c_{0}=0.85-1.37 \mathrm{~mm}\right)$. The stress $(\sigma)-$ elongation ratio $(\lambda)$ curve of the notched sample was measured, whereby $\lambda$ was determined by a video extensometer that measures the displacement of two white dots. These dots were placed as markers on the lower half of the notched sample (Figures S2b and S3a), ${ }^{\mathrm{S} 11}$ and the stress was characterized as the external load $(\mathrm{N})$ divided by the unnotched cross-sectional area in the undeformed state. $\lambda_{\mathrm{c}}$ and $W_{\mathrm{c}}$ were measured from the stress $(\sigma)$ - elongation ratio $(\lambda)$ curve thus obtained.

First, we measured $G_{\mathrm{c}}$ of the virgin DN gel. The typical $\sigma-\lambda$ curves of the notched and unnotched samples are shown in Figure S2c. The notched DN gel ruptured at $\lambda_{\mathrm{c}}=1.84 \pm 0.07$ (number of tests $n=4$ ), while the unnotched sample ruptured at $\lambda$ in the range of $5-8$. The $\sigma-\lambda$ curve of the notched sample almost overlaps (within $10 \%$ of the typical stress) with that of the unnotched sample before rupture. Typically, the input mechanical energy density, $W_{\text {in, }}$ corresponding to the area under the $\sigma-\lambda$ curve, is used for $W_{\mathrm{c}}$ in Equation S1. ${ }^{\mathrm{S} 3 \mathrm{~S} 5}$ When we used $W_{\text {in }}\left(1.7( \pm 0.2) \times 10^{5} \mathrm{~J} \mathrm{~m}^{-3}\right)$, the fracture energy was determined as $G_{\mathrm{c}}=826 \pm 245 \mathrm{~J} \mathrm{~m}^{-2}(n=4)$, which is close to the fracture energy obtained from the tearing test with a trouser-shaped sample $\left(906 \pm 62 \mathrm{~J} \mathrm{~m}^{-2}\right)$. It should be noted that the DN gel showed mechanical hysteresis at $\lambda \gtrsim 1.3$. Some researchers have suggested that $W_{\mathrm{c}}$ in Equation $\mathrm{S} 1$ is the stored elastic energy density, $W_{\mathrm{el}}$, which excludes the dissipated energy density. ${ }^{\mathrm{S}}$ From the cyclic tensile test of the DN gel (Figures S4a and S4c), the stored elastic energy density at an elongation ratio of $\lambda=1.8$ is $W_{\mathrm{el}}=6.4 \times 10^{4} \mathrm{~J} \mathrm{~m}^{-3}\left(40 \%\right.$ of the $\left.W_{\mathrm{in}}\right)$. Note that the stress-strain curve of the DN gels is known 
to be independent of the strain rate and that the hysteresis is irreversible. ${ }^{\mathrm{S} 12, \mathrm{~S} 13}$ Using this $W_{\mathrm{el}}$ in Equation $\mathrm{S} 1$, the fracture energy was determined as $G_{\mathrm{c}}=333 \pm 92 \mathrm{~J} \mathrm{~m}^{-2}$, which is much smaller than the trouser-tearing test result. Herein, we regard the $G_{\mathrm{c}}$ of the virgin DN gels as $\sim 830 \mathrm{~J} \mathrm{~m}^{-2}$ which was obtained using the input energy density $W_{\text {in }}$ because it is close to the trouser-tearing fracture energy. The discussion on which $W_{\text {in }}$ or $W_{\mathrm{el}}$ should be used for dissipative soft materials is beyond the scope of this paper but needs to be investigated in the future.

Second, the $G_{\mathrm{c}}$ of the PAAm single-network gel was characterized as a model of the second network. The as-prepared PAAm gel, which was not immersed in water after gel synthesis, contained $28 \mathrm{wt} . \%$ of the PAAm network and $72 \mathrm{wt} . \%$ of water, determined from the composition of the sample synthesis. On the other hand, the DN gel used in this work at equilibrium swollen state in water contained 2 wt.\% of the PNaAMPS first network, 17 wt.\% of the PAAm second network and $80 \mathrm{wt} . \%$ water. Therefore, we swell the as-prepared PAAm gel (weight $W(\mathrm{~g}))$ using the $0.65 W(\mathrm{~g})$ of water to make the swollen PAAm gel having the PAAm concentration of $17 \mathrm{wt} . \%$, the same as that in the DN gel. The typical $\sigma-\lambda$ curves of the unnotched and notched samples are shown in Figure S2d. From this result, we determined the fracture energy of the PAAm gel as $G_{\mathrm{c}}=184 \pm 21 \mathrm{~J} \mathrm{~m}^{-2}(n=3)$. Note that this gel showed negligible hysteresis in the observation range (Figure S4b).

We also tested the trouser-tearing test of the controlled-swollen PAAm gel (PAAm: 17 wt.\%) for comparison. Some samples (3 samples out of 7) showed steady-state crack propagation; while the crack of the other samples (4 samples out of 7) propagated almost perpendicular to the original crack direction (Figure S5b) probably because the gel is very soft, stretchable, and relatively tough. From the 3 samples that showed the steady-state crack propagation, the tearing fracture energy of the controlled-swollen PAAm gel (PAAm: $17 \mathrm{wt} . \%)$ is characterized as $\Gamma=168 \pm 54 \mathrm{~J} \mathrm{~m}^{-2}(n=3)$, which is close to $G_{\mathrm{c}}\left(184 \pm 21 \mathrm{~J} \mathrm{~m}^{-2}\right)$.

For references, single-edge notch test and tearing test of the as-prepared PAAm gel (PAAm: 28 wt.\%) were also performed. From the single-edge notch test, the fracture energy was characterized as $G_{\mathrm{c}}=343 \pm 78 \mathrm{~J} \mathrm{~m}^{-2}(n=4)$. In the trouser-tearing test, some samples showed steady-state crack propagation (Figure S5a). From the tearing test results, the tearing fracture energy of the as-prepared PAAm gel (PAAm: $28 \mathrm{wt} . \%)$ is characterized to be $199 \pm 14 \mathrm{~J} \mathrm{~m}^{-2}(n=4)$, which is smaller than $G_{\mathrm{c}}\left(343 \pm 78 \mathrm{~J} \mathrm{~m}^{-2}\right)$ but has an agreement with that as an order-magnitude estimation (within a factor of 2).

Third, we measured the $G_{\mathrm{c}}$ of the stretched DN gel, in which plenty of internal fracturing had already occurred (Figure S3). ${ }^{\mathrm{S} 14}$ In this batch of the DN gel, the elongation ratio at the breaking point varied in the range of 5-8. Hence, we imposed an elongation ratio $\lambda$ of 6.0 , which is close to the attainable maximum elongation ratio. The experimental procedure is shown in Figure S3a. First, an unnotched DN gel was stretched to $\lambda=6.0$ to induce internal fracturing, which was then unloaded. The sample returned to almost the original length in the relaxed state (the residual strain was approximately $6 \%$ ). Next, a small notch $(\sim 1 \mathrm{~mm})$ was created on the pre-stretched DN gel. Then, the stretched DN gel with the small notch was 
stretched until rupture. The typical $\sigma-\lambda$ curves are shown in Figure S3b. Note that the stretched DN gel $(\lambda=6)$ shows no hysteresis in the range of $1<\lambda<6$. Using Equation S1, we obtained the fracture energy of the stretched DN gel as $G_{\mathrm{c}}=697$ $\pm 123 \mathrm{~J} \mathrm{~m}^{-2}(n=3)$, which is surprisingly higher than that of the second network $\left(150-200 \mathrm{~J} \mathrm{~m}^{-2}\right)$.

All results of fracture energy of the DN gels (virgin and pre-stretched) and the PAAm SN gels (as-prepared and controlled-swollen) characterized in this work are summarized in Table 1 in the main text. 
(a)

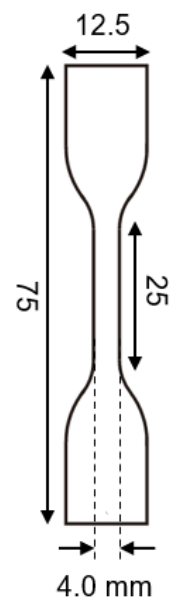

(b)

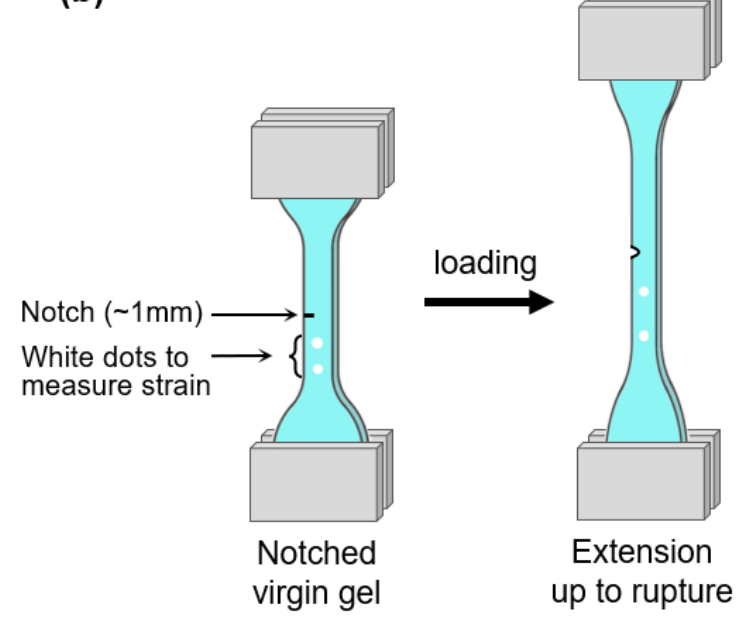

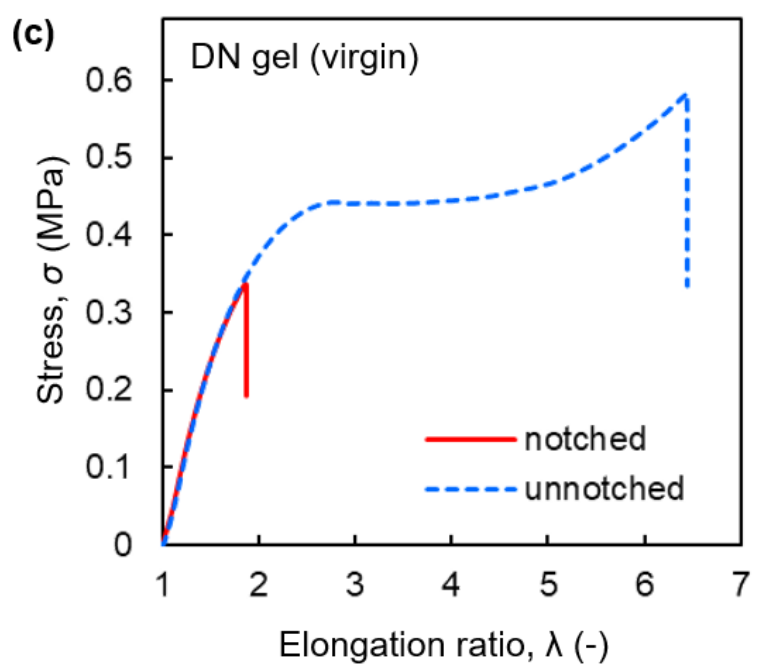

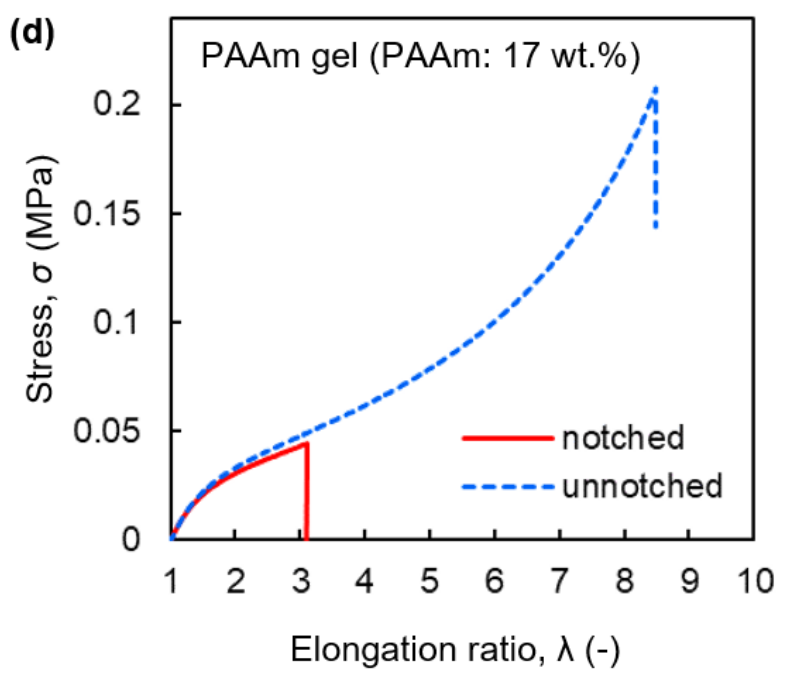

Figure S2. Single-edge notch test for measuring the fracture energy of the virgin DN gel and the controlled-swollen PAAm single-network gel (PAAm: 17 wt.\%). (a) Dumbbell-shaped geometry standardized to JIS-K 6152-6 used in this experiment. (b) Schematic illustration of the fracture test with a small notch. (c), (d) Typical stress-elongation ratio curves of the virgin DN gel and the PAAm gel for notched and unnotched samples. The PAAm SN gel was swollen with a controlled amount of water resulting in PAAm concentration of $17 \mathrm{wt} . \%$ that is the same PAAm concentration in the DN gel. 
(a)
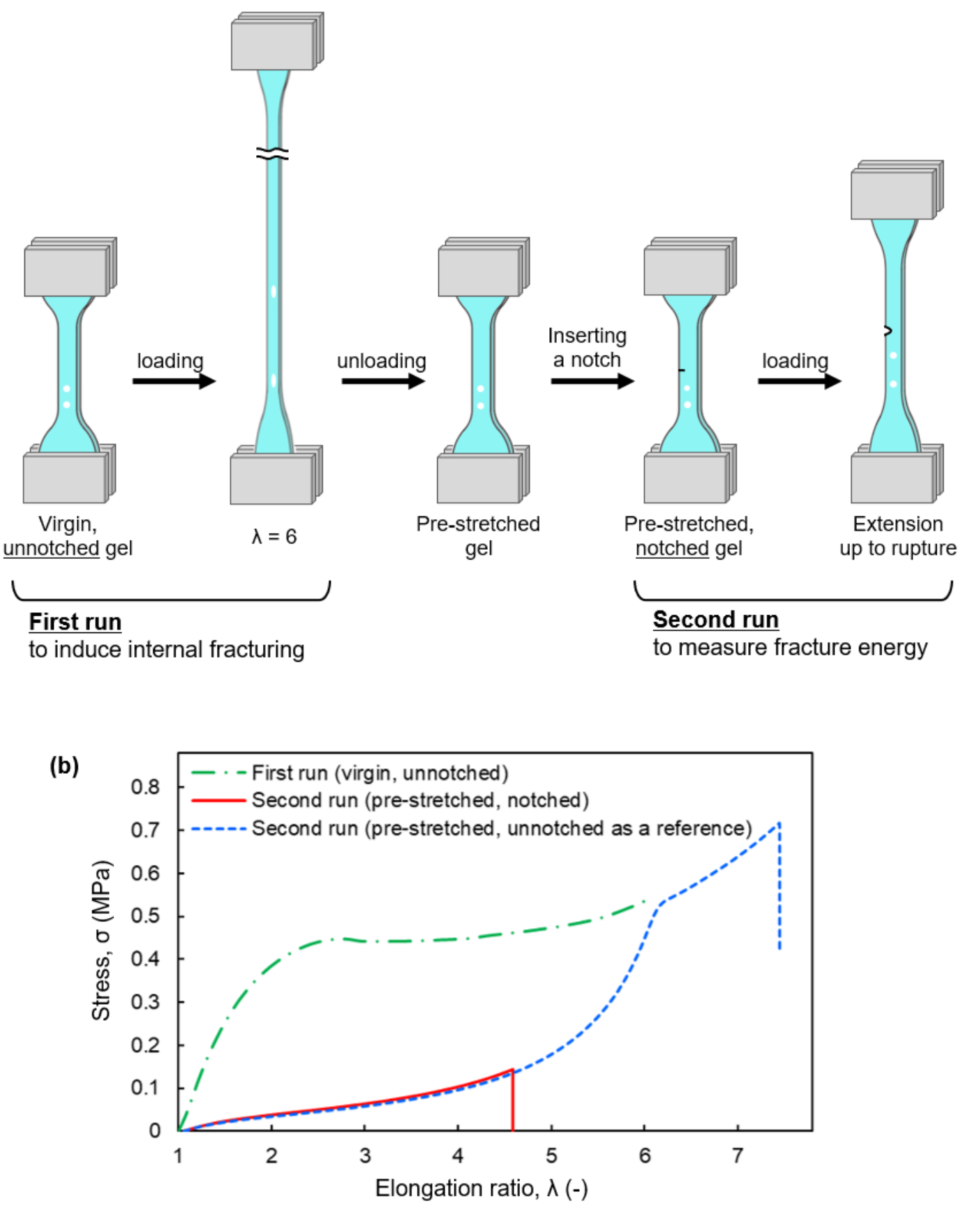

Figure S3. Single-edge notch test for measuring the fracture energy of a pre-stretched DN gel, in which internal fracturing has already occurred. (a) Schematic illustration of the measurement. First, a virgin DN gel without a notch was stretched to the elongation ratio $\lambda=6$ to induce plenty of internal fracturing in the first network (first run), and then, the gel was unloaded. Subsequently, a small notch $(\sim 1 \mathrm{~mm})$ was made on one edge of the sample. Finally, this pre-stretched and notched DN gel was stretched until rupture (second run). (b) Typical stress-elongation ratio curves of the first run (virgin unnotched DN gel) and the second run of the pre-stretched DN gels with and without a notch. 

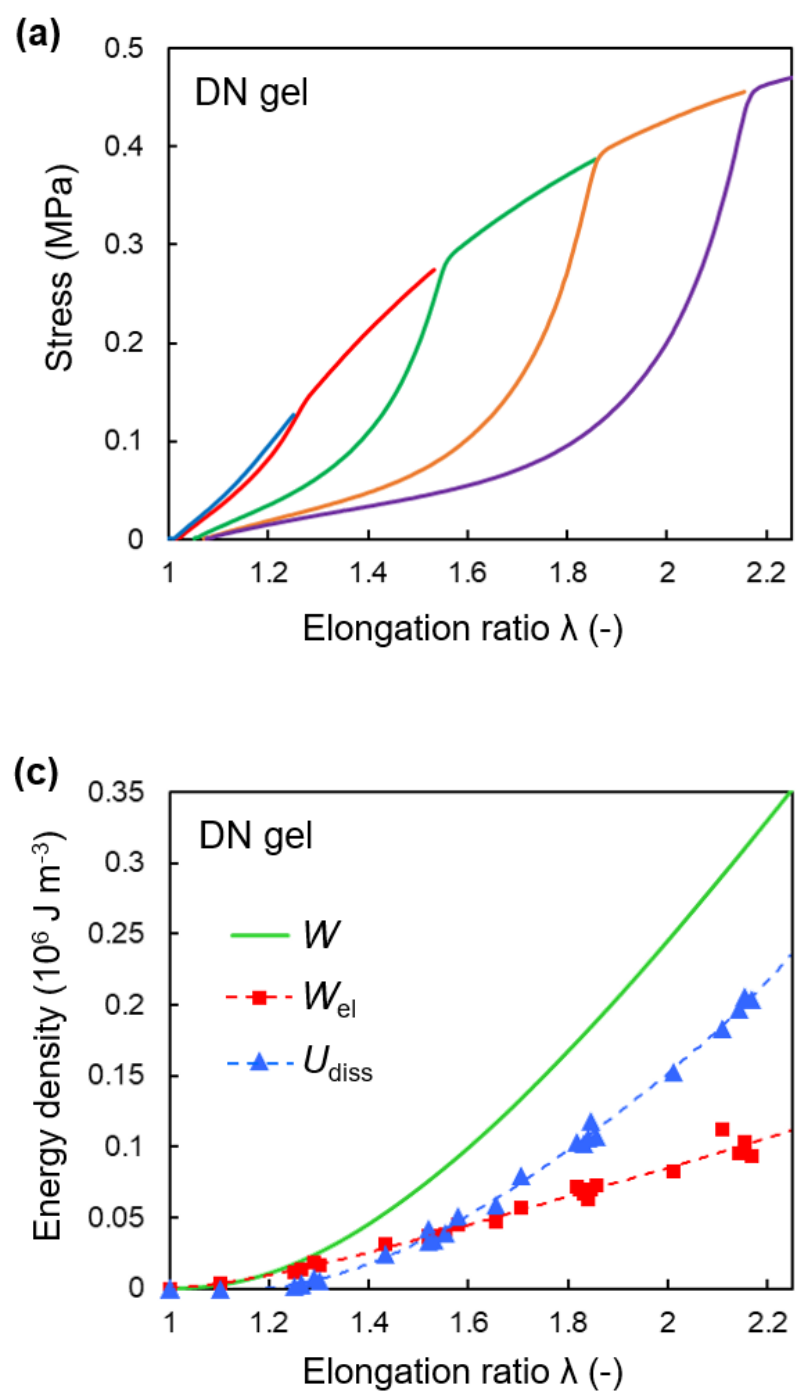

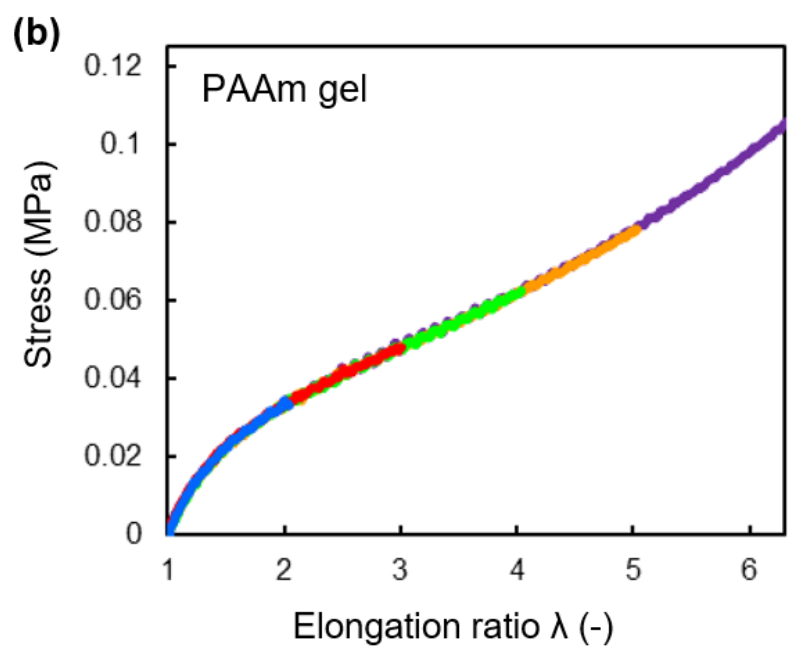

(d)

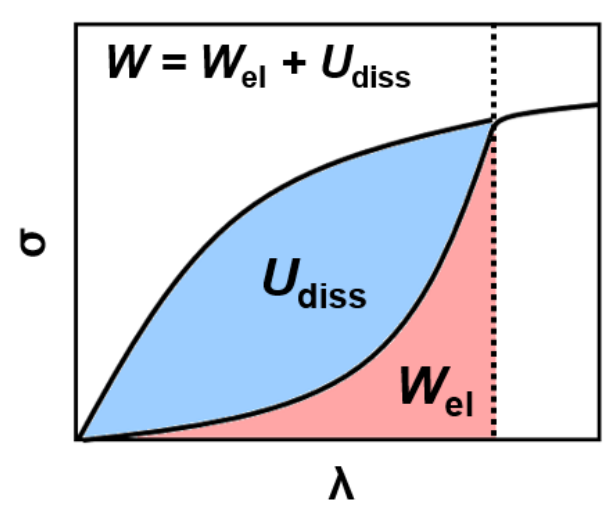

Figure S4. Characterization of mechanical hysteresis and stored elastic energy. (a), (b) Typical stress-elongation ratio loading curves of cyclic tensile test of unnotched samples of (a) the DN gel and (b) the controlled-swollen PAAm gel (PAAm: $17 \mathrm{wt} . \%$ ). The range of interest of $\lambda$ is up to the rupture point of the notched sample, $\lambda_{\mathrm{c}} \approx 2$ for the DN gel and $\lambda_{\mathrm{c}} \approx$ 3 for the PAAm gel. Significant hysteresis appeared in the DN gel due to internal fracturing, whereas no hysteresis was observed in the PAAm gel. (c) Input energy density $W_{\text {in }}$, elastic stored energy density $W_{\mathrm{el}}$ and dissipated energy density $U_{\text {diss }}$ as a function of the elongation ratio $\lambda$ of the $\mathrm{DN}$ gel. $W_{\text {in }}$ was obtained based on the area under a typical $\sigma-\lambda$ curve of the DN gel. The data points of $W_{\mathrm{el}}$ and $U_{\mathrm{diss}}$ represent the data of multiple measurements of this DN gel obtained from cycle tensile test. The dashed lines in (c) are guides for the eyes. (d) Schematic illustration of $U_{\text {diss }}$ and $W_{\mathrm{el}}$. As the DN gel shows irreversible hysteresis, the relation of $W_{\mathrm{in}}=W_{\mathrm{el}}+U_{\text {diss }}$ is satisfied. 
(a) As-prepared PAAm gel (PAAm: 28 wt. \%)

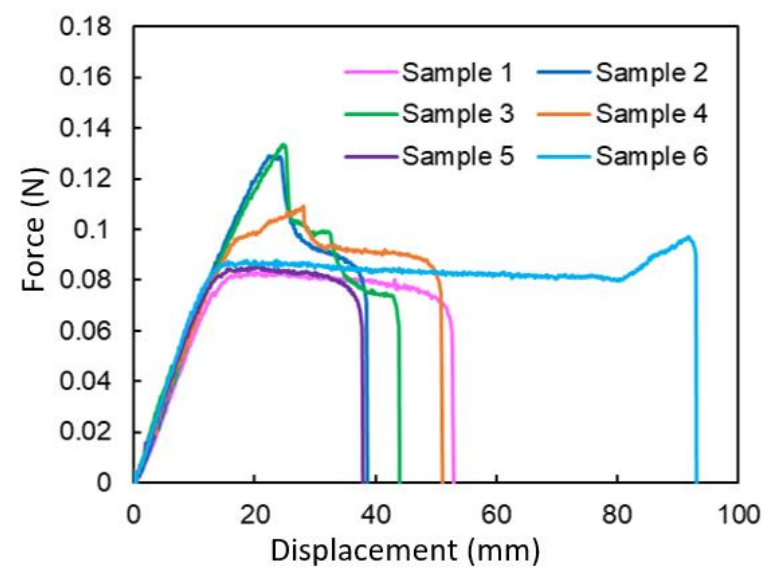

(c) As-prepared, sample 1

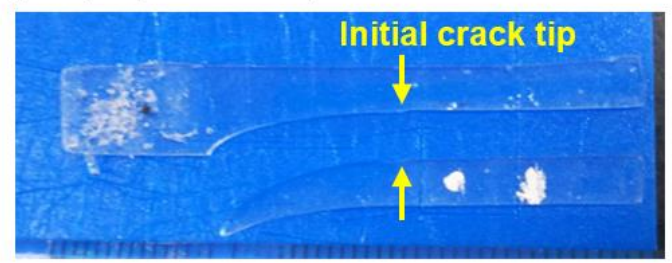

(d) As-prepared, sample 6

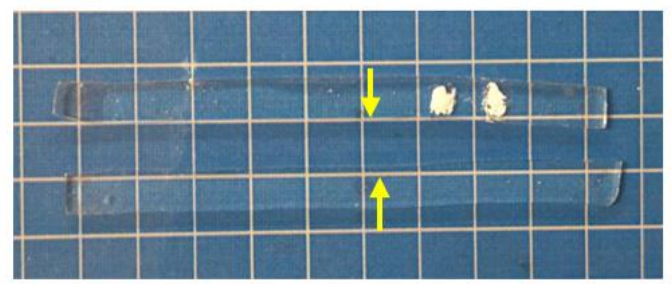

(b) Controlled-swollen PAAm gel (PAAm: 17 wt.\%)

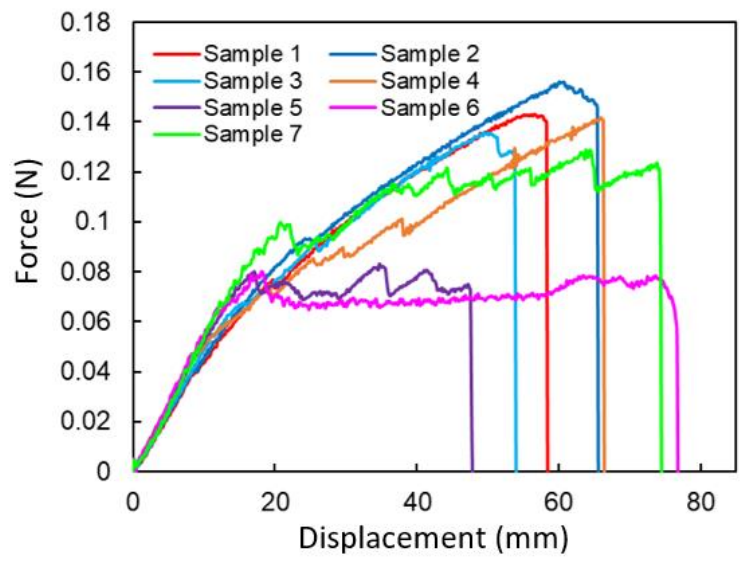

(e) Controlled-swollen, sample 2

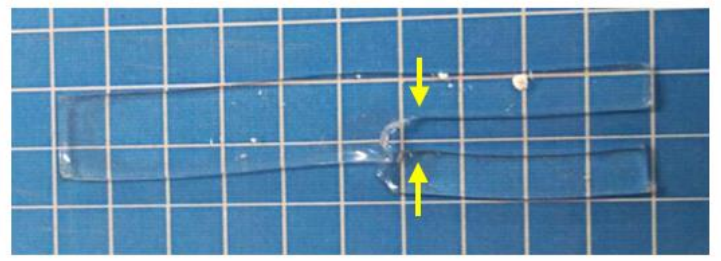

(f) Controlled-swollen, sample 6

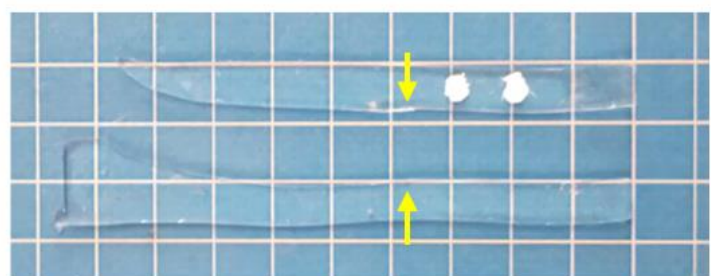

Figure S5. Trouser-shaped tearing fracture test of PAAm single-network gels for references. (a), (b) Force-displacement curves under the tearing test. (a) For the as-prepared PAAm gel (PAAm: 28 wt.\%, thickness $1.0 \mathrm{~mm}$ ), samples 2 and 3 did not show steady-state crack propagation, so that these samples were not used for the fracture energy calculation. From the steady-state regions in samples 1, 4, 5 and 6, the tearing fracture energy was characterized as $\Gamma=199 \pm 14 \mathrm{~J} \mathrm{~m}^{-2}(n=4)$. (b) For the controlled-swollen PAAm gel (PAAm: 17 wt.\%, thickness $1.2 \mathrm{~mm}$ ), samples 1, 2, 3 and 4 did not show steady-state crack propagation. Samples 5, 6 and 7 showed steady-state crack propagation, some of which (samples 5 and 7) showed stick-slip like phenomenon. From the three samples 5, 6 and 7, the tearing fracture energy was characterized as $\Gamma=168 \pm 54$ $\mathrm{J} \mathrm{m}^{-2}(n=3)$. (c)-(f) Pictures of some torn PAAm gels. Yellow arrows indicate the approximate positions of the tip of the initial crack made by a razor blade. (d) On sample 6 of the as-prepared PAAm gel, crack propagation went straight throughout the sample. (c), (f) On sample 1 of the as-prepared PAAm gel and sample 6 of the controlled-swollen PAAm gel, steady-state crack propagation was observed but the propagation gradually deviated from the center part of the sample. (e) On sample 2 of controlled-swollen gel, steady-state crack propagation was not observed and the crack directly went almost perpendicular direction to the initial crack, which is not used for the fracture energy calculation. 


\section{Supporting Note II:}

\section{Potential errors in the fluorescence method using mechanoradical polymerization}

The dissipated energy density profile (Figure $\mathbf{2 d}$ in the main text) characterized by mechanoradical polymerization method combined with fluorescent molecules and laser-scanning confocal microscopy (LSCM) can contain some uncertain method-specific errors. We discuss the potential reasons for errors in this section.

First, in this study, we used the results of a uniaxial tensile test for calibrating data around the crack tip (Figure 2c in the main text). The $I-U_{\text {diss }}$ relation around the crack tip in the tearing test may not be exactly identical to the $I-U_{\text {diss }}$ relation of the uniaxially stretched samples. Deformation around the crack tip is more complicated than simple uniaxial tension, which causes a discrepancy in the $I-U_{\text {diss }}$ relations between uniaxial stretching and tearing. However, we consider the discrepancy to be small for the following reasons. A theory of non-linear elastic solids using the generalized neo-Hookean model implies that deformations close to a crack tip subjected to mode I deformation are dominated by uniaxial tension. ${ }^{\mathrm{S} 15}$ Uniaxial tension close to a crack tip is also expected in the tearing fracture of a DN gel in our test. With an increase in the distance from the crack tip, the uniaxial tension may gradually change to pure-shear tension. Even so, it has been found that the stress in the principal direction and the dissipated energy density of a DN gel under pure-shear deformation are very close (typically less than $10 \%$ difference) to those in uniaxial deformation at the same principal strain (at least for the strain below the yielding point). ${ }^{\mathrm{S} 16}$ In addition, it is expected that the fluorescence intensity directly reflects the number density $\left(\mathrm{mol} \mathrm{m}^{-3}\right)$ of the broken polymer strands, which correlates with the energy dissipation density $\left(\mathrm{J} \mathrm{m}^{-3}\right)$. Therefore, we can approximate the energy dissipation around the crack tip by calibration using the fluorescence intensities of the uniaxially stretched samples. The other potential error between tearing and stretching is the difference in strain rate. The strain rate around the crack tip under tearing was higher than that in the uniaxial tensile test. The kinetic difference induces different rates of mechanoradical generation, which might influence the degree of the mechanoradical polymerization.

Second, we measured $U_{\text {diss }}(z)$ only near the sample surface (i.e., x-z plane at y $\approx 5 \mathrm{~mm}$ ) and assumed in Eq. (4) that the profile $U_{\text {diss }}(z)$ does not change along the sample thickness direction (y-direction, see Figure 2a in the main text for the $(\mathrm{x}, \mathrm{y}, \mathrm{z})$ coordinates). If the actual $U_{\text {diss }}(z)$ varies in the y-direction, $\Gamma_{\text {diss }}$ as calculated from Eq. (4) contains an error.

Finally, water migration may potentially occur. Although we observed that there is no difference in sample thickness between the damaged and undamaged regions, some water migration from the undamaged region to the damaged region may occur because of a difference in the osmotic pressure between the two regions. If this is the case, PNIPAAm in the damage zone is diluted, which reduces the fluorescence intensity. Note that PNIPAAm migration is negligible because PNIPAAm strands are expected to be tethered to the broken ends of the first network. ${ }^{\mathrm{S} 17}$ 
These three factors potentially affect the quantitative results of $U_{\text {diss }}(z)$ and $\Gamma_{\text {diss }}$ to some extent. Therefore, we determined the value of $\Gamma_{\text {diss }}$ using ranges (see Figure 4 in the main text). Although it was difficult to determine the error range only from our results of the fluorescence measurements, the possible range of $\Gamma_{\text {diss }}$ was determined from the values of $\Gamma$ and $\Gamma_{0}$ obtained in separate mechanical tests, using the relation $\Gamma=\Gamma_{\text {diss }}+\Gamma_{0}$. 


\section{References for Supporting Information}

(S1) Rivlin, R. S.; Thomas, A. G. Rupture of rubber. I. Characteristic energy for tearing. J. Polym. Sci. 1953, 10, 291-318.

(S2) Greensmith, H. W. Rupture of rubber. X. The change in stored energy on making a small cut in a test piece held in simple extension. J. Appl. Polym. Sci. 1963, 7, 993-1002.

(S3) Ducrot, E.; Chen, Y.; Bulters, M.; Sijbesma, R. P.; Creton, C. Toughening elastomers with sacrificial bonds and watching them break. Science 2014, 344, 186-189.

(S4) Mayumi, K.; Guo, J.; Narita, T.; Hui, C. Y.; Creton, C. Fracture of dual crosslink gels with permanent and transient crosslinks. Extreme Mech. Lett. 2016, 6, 52-59.

(S5) Guo, H.; Sanson, N.; Hourdet, D.; Marcellan, A. Thermoresponsive toughening with crack bifurcation in phase-separated hydrogels under isochoric conditions. Adv. Mater. 2016, 28, 5857-5864.

(S6) Chen, Y.; Yeh, C. J.; Qi, Y.; Long, R.; Creton, C. From force-responsive molecules to quantifying and mapping stresses in soft materials. Sci. Adv. 2020, 6, eaaz5093.

(S7) Creton, C. 50th anniversary perspective: Networks and gels: Soft but dynamic and tough. Macromolecules 2017, 50, $8297-8316$.

(S8) Long, R.; Hui, C.-Y. Fracture toughness of hydrogels: Measurement and interpretation. Soft Matter 2016, 12, 80698086.

(S9) Lindley, P. B. Energy for crack growth in model rubber components. J. Strain Anal. Eng. Des. 1972, 7, $132-140$.

(S10) Naït-Abdelaziz, M.; Zaïri, F.; Qu, Z.; Hamdi, A.; Hocine, N. A. J integral as a fracture criterion of rubber-like materials using the intrinsic defect concept. Mech. Mater. 2012, 53, 80-90.

(S11) Matsuda, T.; Nakajima, T.; Gong, J. P. Fabrication of tough and stretchable hybrid double-network elastomers using ionic dissociation of polyelectrolyte in nonaqueous media. Chem. Mater. 2019, 31, 3766-3776.

(S12) Na, Y.-H.; Tanaka, Y.; Kawauchi, Y.; Furukawa, H.; Sumiyoshi, T.; Gong, J. P.; Osada, Y. Necking phenomenon of double-network gels. Macromolecules 2006, 39, 4641-4645.

(S13) Webber, R. E.; Creton, C.; Brown, H. R.; Gong, J. P. Large strain hysteresis and Mullins effect of tough double-network hydrogels. Macromolecules 2007, 40, 2919-2927.

(S14) Zhang, T.; Lin, S.; Yuk, H.; Zhao, X. Predicting fracture energies and crack-tip fields of soft tough materials. Extreme Mech. Lett. 2015, 4, 1-8.

(S15) Long, R.; Hui, C. Y. Crack tip fields in soft elastic solids subjected to large quasi-static deformation — A review. Extreme Mech. Lett. 2015, 4, 131-155.

(S16) Mai, T.-T.; Matsuda, T.; Nakajima, T.; Gong, J. P.; Urayama, K. Distinctive characteristics of internal fracture in tough double network hydrogels revealed by various modes of stretching, Macromolecules 2018, 51, 5245-5257.

(S17) Matsuda, T.; Kawakami, R.; Namba, R.; Nakajima, T.; Gong, J. P. Mechanoresponsive self-growing hydrogels inspired by muscle training. Science 2019, 363, 504-508. 